uranium co-exposure. A potential excess risk of lung cancer related to chromium (VI) exposure observed among nuclear workers should be confirmed in further studies.

\section{MORTALITY STUDY IN TWO ITALIAN COHORTS OF WORKERS EXPOSED TO PCBS: EXTENSION OF THE FOLLOW-UP UP TO 2006}

${ }^{1} \mathrm{~A}$ C Pesatori, ${ }^{2}$ Grillo, ${ }^{2}$ Consonni, ${ }^{3}$ Caironi, ${ }^{3}$ Sampietro, ${ }^{1}$ Bertazzi. ' University of Milan, Milan, Italy; ${ }^{2}$ IRCCS Foundation, Ospedale Maggiore Policlinico, Milan, Italy; ${ }^{3}$ Local Health Unit (ASL), Bergamo, Italy

10.1136/oemed-2013-101717.247

Objectives PCBs have been classified as carcinogenic in animals and probably carcinogenic to humans (IARC1987). Epidemiological studies showed increased risks for Non-Hodgkin's Lymphoma, liver and biliary tract, digestive, skin, prostate and brain cancer but findings are still controversial. We present the results of the extension of the follow-up as of 2006 of two cohorts of workers potentially exposed to PCBs in capacitor manufacturing plants in Lombardy, Northern Italy. The mortality of the two cohorts was previously examined up to 1991 (Plant 1) and 2002 respectively (Plant 2 ).

Methods The study population included 544 males and 1551 females employed between 1946 and 1978 in Plant 1 and 373 males and 97 females employed between 1950 and 1988 in plant 2. Standardised mortality ratios (SMR) and 95\% Confidence Intervals $(95 \% \mathrm{CI}$ ) were calculated (in the whole cohort, separately in plant 1 and 2 and among males and females) using as reference the regional mortality rates specific by age and calendar period.

Results In the whole cohort, increased mortality from biliary tract and "other digestive" cancer, prostate, brain cancer, Hodgkin and non-Hodgkin lymphoma (8 deaths, SMR 1.65; 95\% CI $0.8-3.3)$ were detected. The excess mortality from biliary tract cancer was confined to males (4 deaths, SMR 3.9; 95\% CI 1.510.4), where as the increased mortality from Hodgkin lymphoma (4 deaths, SMR 3.6, 95\% CI 1.3-9.5) and brain cancer (7 deaths, SMR 2.1; 95\% CI 1.02-4.5) was observed in plant 1 only. The excess mortality from other digestive cancer and NHL was equally distributed in the two cohorts and among males and females. Analyses by duration of employment and time since first employment did not show consistent trends.

Conclusions Our findings suggest increased mortality from cancers of "a priori" interest in PCB exposed workers, however these excesses were not clearly related to duration of employment and latency.

\section{OCCUPATIONAL EXPOSURE TO TRICHLOROETHYLENE AND PERCHLOROETHYLENE AND THE RISK OF LYMPHOMA, LIVER AND KIDNEY CANCER IN FOUR NORDIC COUNTRIES}

\begin{abstract}
${ }^{1} \mathrm{~J} J$ Vlaanderen, ${ }^{1}$ Straif, ${ }^{2}$ Pukkala, ${ }^{3}$ Kauppinen, ${ }^{4}$ Kyyronen, ${ }^{5}$ Martinsen, ${ }^{5}$ Kjaerheim, ${ }^{6}$ Tryggvadottir, ${ }^{7}$ Hansen, ${ }^{8}$ Sparen, ${ }^{9}$ Weiderpass. ${ }^{1}$ IARC, Lyon, France; ${ }^{2}$ School of Public Health, University of Tampere, Tampere, Finland; ${ }^{3}$ Finnish institute of occupational health, Helsinki, Finland; ${ }^{4}$ Finnish cancer registry, Helsinki, Finland; ${ }^{5}$ Cancer registry of Norway, Oslo, Norway; ${ }^{6}$ Faculty of Medicine, University of Iceland, Reykjavik, Iceland; ${ }^{7}$ Institute of Cancer Epidemiology, Danish cancer society, Copenhagen, Denmark; ${ }^{8}$ Department of Medical Epidemiology and Biostatistics, Karolinska Institute, Stockholm, Sweden; ${ }^{9}$ Folkhalsan Research Center, Samfundet Folkhasan, Helsinki, Finland
\end{abstract}

10.1136/oemed-2013-101717.248
Objectives Trichloroethylene (TCE) and Perchloroethylene (PER) are two chlorinated solvents that have been applied widely as degreaser of metal parts and in dry cleaning and other applications. In 2012 the International Agency for Research on Cancer classified TCE as carcinogenic to humans and PER as probably carcinogenic to humans. We explored exposureresponse relations for TCE and PER and non-Hodgkin lymphoma (NHL), multiple myeloma (MM), and cancers of the kidney and liver in the Nordic Occupational Cancer (NOCCA) cohort which holds information on occupation and long-term follow-up for cancer for the populations of Norway, Sweden, Finland and Iceland.

Methods The cohort was set-up by linking occupational information from censuses to national cancer registry data utilising personal identity codes in use in all the Nordic countries. Country, time-period, and job-specific exposure estimates were generated for TCE, PER, and potentially confounding occupational exposures with a comprehensive job-exposure matrix. Two exposure metrics were created: cumulative exposure and 'average intensity times prevalence' of exposure. Conditional logistic regression was conducted for exposure groups as well as for continuous cumulative exposure.

Results Hazard ratios for liver cancer, NHL, MM, but not kidney cancer were slightly elevated in groups with high exposure to PER (compared to occupationally unexposed subjects). Hazard ratios for liver cancer and NHL also increased with increasing continuous exposure to PER. We did not observe evidence for an association between exposure to TCE and NHL, MM, and liver and kidney cancer.

Conclusions Although this study was subject to limitations related to the low prevalence of exposure to PER and TCE in the Nordic population and a necessarily rudimentary exposure assessment strategy, we observed some evidence indicative of an excess risk of cancer of the liver and NHL in subjects exposed to PER. Exposure misclassification may have diluted the observed associations in this study.

\section{A COMPREHENSIVE DANISH OCCUPATIONAL CANCER LINKAGE}

J H Hansen, Meersohn. Danish Cancer Society, Copenhagen, Denmark

\subsection{6/oemed-2013-101717.249}

Objectives Linkages of cancer and occupation provide an important resource to aid in our understanding of the role of occupational exposures in the aetiology of cancer. The majority of existing surveys have, however, been based on census data including occupational information from only one certain date, thus lacking the general situation with several changed between different jobs during a work career. We report on a series of 60 Danish nested case-control studies based on nationwide data linkages, including information on all employments since 1964 .

Methods All cancer cases $(\mathrm{N}=1,031,504 ; 50.4 \%$ women and 49.6\% men) diagnosed between 1970 and 2010, aged 18 and 84 years old, was retrieved from the Danish Cancer Registry. Each subject was individually linked with employment information from the Nationwide Pension Scheme with compulsory membership, including information on start and end of each employment, and a 5-digit hierarchically trade code. Information on job title, civil and vital status was obtained from the Central Population Register (CPR). Controls free of cancer and matched on birth year and sex have been selected from the 
$\mathrm{CPR}$ and linked in the same way as cases. Finally, individual information on SES, residential history, reproduction, prescribed medicine, and comorbidity has been added to each case and control. Finally, a JEM on potential carcinogens can be applied.

Results Results confirm increased risk for e.g. lung and bladder cancer among painters and for nasal cancer in wood dust exposed workers. On the other hand, farmers, gardeners and forestry workers had deficits for many cancers. Results on new significant associations will also be presented.

Conclusions These data confirms many well established associations between work and cancer, and demonstrates that many associations are not fully explained. The large number of cancers available for analysis provides the opportunity to evaluate possible occupational associations even with rare cancers.

\section{LONG-TERM EFFECTS OF EXPOSURES AT OAK RIDGE NATIONAL LABORATORY}

B Richardson, Wing, Keil, Wolf. University of North Carolina, Chapel Hill, United States of America

\subsection{6/oemed-2013-101717.250}

We examine mortality in a cohort of workers at the Oak Ridge National Laboratory (ORNL), a US Department of Energy research and development facility. The last mortality follow-up of this epidemiologically important cohort was conducted 18 years ago and included workers hired between 1943 and 1972. Analyses of these data revealed a positive association between occupational radiation dose and all cancer mortality under a 20year lag assumption $(1.73 \%$ increase in all cancer mortality per $10 \mathrm{mSv}$, standard error $=0.86$ ). We have expanded this cohort to include 22,834 workers hired between 1943 and 1985, and updated mortality follow-up through 2008, yielding almost three times the number deaths observed in the last mortality follow-up of an ORNL cohort. Extending follow-up of this cohort is important not just for statistical power, but also because many important questions in radiation research concern the long term effects of irradiation, particularly on malignant diseases. These workers were individually badge-monitored for external exposure to ionising radiation, allowing evaluation of the effects of protracted radiation exposures accrued at work. We compared the observed deaths to expectations based upon US mortality rates, and evaluated radiation dose-mortality associations. Findings include excess deaths due to cancer of the pleura $(\mathrm{SMR}=$ 12.09 95\%CI: 4.44, 26.32), cancer of the bladder (SMR $=1.89$ 95\%CI: 1.26, 2.71), and leukaemia (SMR $=1.33$ 95\%CI: 0.87, 1.93) among hourly-paid males, and excess deaths due to cancer of the bladder (SMR $=2.20$ 95\%CI: 1.20, 3.69) and leukaemia $(\mathrm{SMR}=1.64$ 95\%CI: 1.09, 2.36) among females. Further results will be presented.

\section{Session: 5. Muscoskeletal disorders}

\section{DIFFERENCES IN THE DETERMINANTS OF EXTENSIVE AS COMPARED WITH MORE LIMITED MUSCULOSKELETAL PAIN}

D Coggon, G Ntani, K T Palmer. University of Southampton, Southampton, United Kingdom

10.1136/oemed-2013-101717.251
Objectives To explore whether multi-site musculoskeletal pain differs from more localised musculoskeletal pain in its association with risk factors.

Methods As part of the CUPID study, standardised questionnaires were used to ascertain exposure to risk factors and the prevalence of pain for a day or longer during the past month at each of 10 anatomical sites. Analysis was based on 12,410 participants from 47 occupational groups (mostly nurses and office workers) in 18 countries. Associations with risk factors were assessed by Poisson regression and summarised by prevalence rate ratios (PRRs).

Results Extensive pain (i.e. at six or more of the 10 anatomical sites examined) was much more prevalent than would have been expected by chance coincidence. In comparison with limited pain (i.e. at 1-3 sites), extensive pain showed distinctive associations with demographic characteristics and a much stronger relation to somatising tendency (PRR 4.6, 95\% CI 3.5-6.1 v 1.3, 95\% CI 1.2-1.4) and reported heavy physical loading (PRR 5.0, 95\% CI 2.8-9.2 v 1.4, 95\% CI 1.2-1.6). It also varied differently between occupational groups. Thus, for example, nurses in Spain had the highest rate of limited pain $(64.3 \%)$, but one of the lowest rates of extensive pain (3.5\%). In contrast, the prevalence of extensive pain among office workers in Nicaragua was $14.0 \%$, whereas that of limited pain was only $40.4 \%$.

Conclusions Extensive musculoskeletal pain has different determinants from pain affecting fewer anatomical sites. Its prevalence varies substantially between occupations and countries, and in a different way from limited pain. In research on causes of pain at specific anatomical sites, it may be important to distinguish cases with pain only at the site of interest from those with pain also at multiple other sites.

\section{DISABLING MUSCULOSKELETAL PAIN IN WORKING POPULATIONS: IS IT THE JOB THE PERSON OR THE CULTURE?}

D Coggon, G Ntani, K T Palmer. University of Southampton, Southampton, United Kingdom

\subsection{6/oemed-2013-101717.252}

Objectives To compare the prevalence of disabling low back pain (DLBP) and disabling wrist/hand pain (DWHP) among groups of workers carrying out similar physical activities in different cultural environments, and to explore explanations for observed differences.

Methods Standardised questionnaires were used to ascertain pain that interfered with everyday activities and exposure to possible risk factors in 12,426 participants from 47 occupational groups (mostly nurses and office workers) in 18 countries. Associations with risk factors were assessed by Poisson regression.

Results The one-month prevalence of DLBP in nurses varied between countries from $9.6 \%$ to $42.6 \%$, and that of DWHP in office workers from $2.2 \%$ to $31.6 \%$. Rates of disabling pain at the two anatomical sites co-varied $(r=0.76)$, but DLBP tended to be relatively more common in nurses and DWHP in office workers. Established risk factors such as occupational physical activities, psychosocial aspects of work and tendency to somatise were confirmed, and associations were found also with adverse health beliefs and group awareness of people outside work with musculoskeletal pain. However, after allowance for these risk factors, up to eightfold differences in prevalence remained. Systems of compensation for work-related illness, and financial 